\title{
UBICACIÓN Y DIMENSIONAMIENTO DE GENERACIÓN DISTRIBUIDA: UNA REVISIÓN
}

\section{LOCATION AND SIZING OF DISTRIBUTED GENERATION: A REVIEW}

\author{
Luis Fernado Grisales* \\ Bonie Johana Restrepo Cuestas** \\ Fredy Esteban Jaramillo***
}

Fecha de recepción: 19 de octubre de 2016

Fecha de revisión: 20 de febrero de 2017

Fecha de aprobación: 3 de mayo de 2017

Cómo citar: L. F. Grisales, B. J. Restrepo Cuestas y F. E. Jaramillo, "Ubicación y dimensionamientos de generación distribuida: una revisión," Ciencia e Ingeniería Neogranadina, vol. 27, no. 2, pp. 157-176. DOI: http://dx.doi.org/10.18359/rcin.2344

\section{RESUMEN}

La generación distribuida (GD) se presenta como un apoyo para suplir la demanda del sistema de forma localizada, lo que permite además como alternativas de generación el uso de fuentes de energía renovables a pequeña escala. Como aporte principal, este artículo presenta las consideraciones que se deben tener en cuenta al momento de analizar, modelar y solucionar el problema de ubicación y dimensionamiento de GD en la red eléctrica de distribución. Inicialmente, se enuncian los aspectos técnicos de la red, el modelado de las cargas e impactos de la GD; seguido de la formulación del modelo matemático del problema, principales técnicas de solución y metodologías aplicadas en la literatura; finalmente, se muestran las investigaciones futuras en este campo de estudio. Todo lo anterior en busca

\footnotetext{
* Ingeniero electricista y magíster en Ingeniería. Docente ocasional e innvestigador del grupo MATyER, del Instituto Tecnológico Metropolitano, Medellín, Colombia. Correo electrónico: luisgrisales@itm.edu.co. ORCID: http://orcid.org/0000-0002-1409-9756

** Ingenieria electricista y magíster en Ingeniería. Docente ocasional e investigadora del grupo MATyER, del Instituto Tecnológico Metropolitano, Medellín, Colombia. Correo electrónico: bonierestrepo@itm.edu.co. ORCID: http://orcid.org/0000-0001-5276-1651

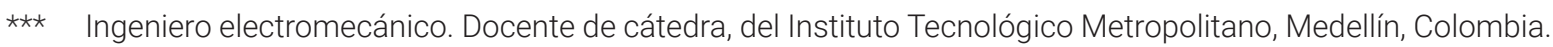
Correo electrónico: fredyjaramillo97913@correo.itm.edu.co. ORCID: http://orcid.org/0000-0001-7878-6291
} 
de mejorar las condiciones operativas de la red. Dentro de la revisión de los trabajos presentados por los diferentes autores, se encontró que una gran parte de los estudios se enfocan en los aspectos técnicos, sin tener en cuenta los costos asociados a la integración de GD, ni los incentivos económicos a los que puede ser susceptibles este tipo de proyectos, que son de vital importancia para evaluar la viabilidad de la propuesta de integración.

Palabras claves: Generación distribuida, sistema de distribución, técnicas de optimización, perfiles de tensión, pérdidas de energía, técnicas metaheurísticas.

\section{ABSTRACT}

Distributed generation (DG) is presented as a support to supply the demand of the system in a localized way, also allowing small-scale generation with renewable energy sources. As a main contribution, this document presents several considerations to be taken into account when analyzing, modeling and solving the problem of location and dimensioning of DG in the distribution grid. Initially, the technical aspects of the network are outlined as well the load modeling, and DG impacts; followed by the formulation of the mathematical model of the problem, main solution techniques, methodologies applied in the literature. Finally, it shows future research in this field of study All the above to improve the operating conditions of the network. Within the review of the papers presented by the different authors, it was found that a large part of the studies focus on the technical aspects, without taking into account the costs associated with the integration of DG, nor the economic incentives that can Be susceptible to this type of projects, which are of vital importance in assessing the feasibility of the integration proposal.

Keywords: Distributed generation, distribution system, optimization techniques, voltage profiles, power losses, metaheuristic techniques.

\section{INTRODUCCIÓN}

La generación de electricidad se presenta generalmente en centrales a gran escala, utilizando tecnologías convencionales en lugares alejados al consumidor final. Esto genera pérdidas de energía asociadas al transporte y alteración en los perfiles de tensión a nivel de distribución, en algunos casos por fuera de los rangos permitidos. Es por esto que la generación distribuida
(GD) se ha convertido en un apoyo a los sistemas de generación convencionales, hecho que facilita la inyección de potencia en lugares cercanos a la carga. No obstante, la integración de GD genera impactos en el sistema, que pueden ser positivos o negativos, debido a que su implementación modifica el modo de operación de la red. Una integración adecuada de GD presenta desde el punto de vista técnico 
los siguientes beneficios: disminución de pérdidas de energía, mejoras en el perfil de tensión y descongestión en las líneas de distribución. Sin embargo, una ubicación o dimensionamiento inapropiado de la GD en el sistema puede generar efectos adversos en la red [1]-[3].

Lo anterior, sumado a la necesidad de aumentar la calidad y confiabilidad del servicio a través de la optimización de recursos y sostenibilidad ambiental, hace que los sistemas de distribución tiendan a convertirse en redes inteligentes (Smart Grids) [4]. Es allí donde la integración de GD ha recibido un creciente interés, ya que puede contribuir en la obtención de resultados en materia de una gestión más activa de la red, reducción de pérdidas de energía, emisiones y la integración de fuentes de energías renovables [1]. En este sentido, algunos países han desarrollado políticas en busca de promover e incentivar la integración de nuevas fuentes de energía dentro de sus sistemas eléctricos. Se pueden mencionar casos como el de los Estados Unidos, con el consorcio de tecnologías para la solución de la confiabilidad eléctrica [5]; la Unión Europea, con su proyecto de microredes [6], y Japón, que es un país con un alto número de proyectos de inclusión de GD, financiados por la Organización para el Desarrollo Nuevas Energías y Tecnología Industrial [7]. Con respecto a Colombia, la Unidad de Planeación Minero Energética (UPME), a través de la Resolución 18-919 de 2010, el convenio ATN/FM12825-CO y la Ley 1715 [8], [9], promueve la creación de marcos legales asociados al uso de las fuentes no convencionales de energía y herramientas que fomenten el uso racional de la energía. Esto además incen- tiva a las universidades, los grupos de investigación y sectores privados a investigar alrededor de estas temáticas, patrocinando proyectos a través de Colciencias. Este aspecto demuestra la necesidad de generar soluciones para integrar sistemas de GD, empleando fuentes convencionales y no convencionales de energía.

Como aporte principal, este documento presenta las consideraciones por tener en cuenta al momento de analizar, modelar y solucionar el problema de integración de GD. Se parte con el numeral 1.1, con una descripción del sistema eléctrico de distribución, haciendo énfasis en los modelos de representación de cargas eléctricas en el sistema, seguido de una definición de GD y la descripción de los impactos que puede presentar en el sistema de distribución, en el numeral 1.2. Luego se muestra en el numeral 2.1 el planteamiento del modelo del problema, que puede ser representado a través de funciones objetivo y el conjunto de restricciones que lo limitan. Adicionalmente, se muestran las técnicas de optimización más empleadas en la integración de GD; de esta manera, se permite al lector comprender, interpretar y formular el problema, seleccionando el tipo de técnica que más se adapte a los factores que desee impactar en el sistema de distribución (SD). Vale la pena destacar que la selección de artículos que contienen la información y metodologías empleadas para integración de GD, fueron realizadas de manera que permitan visualizar un panorama general de los enfoques, métodos y técnicas empleados por los autores en la última década. 


\section{GENERACIÓN DISTRIBUIDA}

\subsection{Sistema eléctrico de distribución}

Un sistema eléctrico de distribución es la parte comprendida entre la subestación eléctrica de media tensión y los usuarios finales. El sistema está conformado por alimentadores, subestaciones de transformación, líneas y cargas, conectados a través de nodos. La variación en las tensiones nodales y en las corrientes a través de las líneas depende de la variación de las cargas. Estas últimas, en un sistema eléctrico, se definen como la representación de la demanda de energía en un nodo [10], dicha representación puede estar asociada a un periodo específico, como la demanda pico (mono-nivel), o tener en cuenta la variación de la curva de demanda diaria del sistema (multi-nivel) [11].

Al analizar el estado operativo del sistema, es necesario tener en cuenta el tipo de carga para la interpretación de los diferentes indicadores del sistema, como las pérdidas de energía por transporte, violación de los perfiles de tensión, índice de estabilidad de tensión, cargabilidad en las líneas, entre otros $[12,13]$. Si se trabaja con una carga mono-nivel se tendrán niveles o aproximaciones para una hora específica, que permiten estimar su estado operativo, pero dentro de un panorama limitado (para la hora cuando se realizó el análisis). Caso contrario, cuando se trabaja con sistemas multi-nivel, que tienen en cuenta el comportamiento de la carga en un margen de 24 horas.

Para conocer el estado operativo del sistema, se aplica el equivalente monofásico del sistema de distribución trifásico [14], y a través de un flujo de carga se obtienen las tensiones nodales y corrientes de línea; variables con las que se definen todos los indicadores del sistema. Vale la pena destacar que con la variación de las demandas nodales (curva de demanda horaria) o la inyección de potencia activa o reactiva en cualquier nodo del sistema, el estado operativo varía drásticamente.

Los valores que tomen las cargas para ambos casos pueden ser obtenidos a través de modelos determinísticos o estocásticos. Para el primer caso, los valores de carga pueden ser seleccionados a través de la creación de curvas de demanda a partir de las cargas nodales en los diferentes sistemas de prueba [15]. Para el segundo caso, que es el más complejo, se parte de un análisis del comportamiento del sistema en un periodo, y se encuentra así el tipo de distribución de probabilidad que mejor represente el problema; este es conocido como incertidumbre en la demanda [16].

\subsection{Definición e impactos de la generación distrinuida}

En los últimos años, el planeamiento de los SD ha buscado mejorar las características operativas del sistema, utilizando estrategias como la reconfiguración de alimentadores, la ubicación de capacitores, los reguladores de tensión, la generación distribuida, entre otros [17]-[19]. La integración de GD es la de mayor investigación a nivel mundial, debido a que permite el uso de fuentes convencionales y no convencionales de energía en el sistema eléctrico, con lo cual se logra para el caso de las energías renovables reducir las emisiones de $\mathrm{CO}_{2}[10]$. 
El concepto de GD no cuenta con una definición generalizada, pero finalmente todas convergen en tres características principales: conexión cercana a la carga, generación a pequeña, mediana y gran escala, y la posibilidad de estar o no conectada a la red de distribución. Según [20], la GD es una fuente de potencia eléctrica conectada directamente a la red de distribución o lo más cercano al medidor del usuario final. La Agencia Internacional de Energía (IEA, por sus siglas en inglés) define GD como una planta de generación que transfiere energía en sitio al consumidor o que sirve de apoyo al SD conectado a la red a niveles de voltaje de distribución [21]. Adicionalmente, las tecnologías de generación basadas en energías renovables presentan las siguientes características: no tienen un despacho centralizado debido a su generación variable y son altamente dependientes de las condiciones climáticas [22]. Desde el punto de vista de la capacidad de generación, se pueden clasificar, según [20], como: micro (generadores desde $1 \mathrm{~W}$ hasta $5 \mathrm{~kW}$ ), pequeña (generadores desde $5 \mathrm{~kW}$ hasta $5 \mathrm{MW}$ ), mediana (generadores desde $5 \mathrm{MW}$ hasta $50 \mathrm{MW}$ ) y grande (generadores desde $50 \mathrm{MW}$ hasta $300 \mathrm{MW}$ ).

Existen cuatro tipos de inyección de potencia por parte de la GD. El tipo uno tiene en cuenta únicamente la inyección de potencia activa; el tipo dos solo considera la inyección de potencia reactiva por parte del generador; el tipo tres considera la inyección tanto de potencia activa, como reactiva a través del generador, y el tipo cuatro permite la inyección de potencia activa por parte del generador y la inyección de potencia reactiva por medio de capacitores, que toman los reactivos de la red para después inyectarlos.
Vale la pena destacar que el tipo más implementado es el 1 , y se modela en el sistema como una carga negativa [23].

La integración de GD puede presentar impactos positivos y negativos en la red. Los impactos positivos son llamados beneficios de apoyo al sistema, dentro de los que se pueden encontrar mejoras en los perfiles de tensión, reducción de pérdidas de energía en la red (debido al transporte) y descongestión de las líneas. Sin embargo, una ubicación o dimensionamiento inapropiado de la GD en el sistema puede generar problemas técnicos, como perfiles de tensión por fuera de los rangos permitidos, fluctuaciones de tensión y violación de los límites de capacidad en las líneas [24]-[27]. En [28] se presentan sus principales desventajas, entre las cuales se encuentran el aumento en los niveles de falla debido a la generación intermitente, los flujos bidireccionales, la coordinación de protecciones (que están diseñadas para flujos unidireccionales), congestiones en la red y fluctuaciones de voltaje (variaciones en el índice de estabilidad). Lo anterior puede afectar tanto la seguridad, como la calidad del suministro eléctrico y debe ser tenido en cuenta al momento de integrar estos dispositivos en el sistema.

Las limitaciones asociadas a la integración de GD pueden ser superadas a través de un correcto dimensionamiento y una ubicación óptima. La evaluación del impacto de GD en un SD se puede realizar a través de un análisis de los flujos de potencia o flujo de carga, con lo cual es posible verificar que la configuración de generadores seleccionada mejore las condiciones operativas del sistema. Dentro de los métodos más 
utilizados en la literatura para calcular el flujo de carga se encuentran: Gauss-Seidel, Newton Raphson, barrido iterativo, entre otros [29]. A partir de las variables entregadas por el flujo de carga, es posible evaluar todos los indicadores del sistema (pérdidas de energía, índice de estabilidad de tensión, factor de potencia, etc.).

Es importante tener en cuenta que la ubicación presenta características binarias, dada la posibilidad de un nodo a ser o no candidato a la instalación de un generador. Mientras que el dimensionamiento es de tipo continuo, por la posibilidad que tiene el generador de tomar un valor de un rango determinado de potencia. Adicionalmente, la no linealidad del sistema de ecuaciones que representa el problema hace que este sea del tipo no lineal entero mixto, y por tanto requiera la implementación de técnicas o paquetes de optimización que permitan obtener una solución adecuada.

\section{UBICACIÓN Y DIMENSIONAMIENTO ÓPTIMO DE GENERACIÓN DISTRIBUIDA}

El uso de una metodología basada en técnicas de optimización debe ser capaz de analizar la influencia o el impacto de la GD sobre las características del SD, que puede ser muy útil en el proceso de planeación y operación de la red [30], [31]. Para encontrar la solución del problema aquí planteado, se deben modelar matemáticamente las condiciones operativas que se desean mejorar y el conjunto de restricciones atadas a ellas, para luego implementar la técnica de optimización seleccionada. A continuación, se presentan los componentes principales para la ubicación y el dimensionamiento de GD, las técnicas de optimización más utilizadas y el análisis de un conjunto de trabajos encontrados en la literatura.

\subsection{Modelado del problema}

En los párrafos anteriores se han definido las condiciones técnicas que se logran mejorar al momento de integrar GD, las cuales se pueden convertir en funciones objetivo. Estas, en conjunto con el sistema de restricciones, forman el modelo matemático del problema, como se muestra en las ecuaciones (1) y (2):

$$
\text { Max o Min } f(x) \rightarrow \text { función objetivo }
$$

Conjunto de restricciones

Una función objetivo es un criterio expresado a través de las variables que definen el comportamiento del sistema [32]. Esta función puede ser maximizada o minimizada según las necesidades del problema. Para el caso de la integración de GD, es posible que pertenezca a los aspectos técnicos o económicos. Las funciones objetivo serían las siguientes:

- Mono-objetivo: se define desde un criterio que busca maximizar o minimizar una sola función objetivo. Las principales funciones mono-objetivo utilizadas para ubicación y dimensionamiento óptimo de GD en el SD son: minimización de pérdidas de energía en el sistema, mini- 
mización de costos de inversión y operativos, minimización de las variaciones en los perfiles de tensión, maximización de la capacidad de generación de GD, maximización de la relación costo/beneficio y maximización del límite de cargabilidad de las líneas [33].

- Multi-objetivo: se define teniendo en cuenta un criterio que busca maximizar o minimizar dos o más funciones objetivo, siempre en conflicto. Las técnicas de optimización que implementa este tipo de funciones entregan un conjunto de soluciones óptimas, conocido como frente óptimo de Pareto [34]. Para la selección de alguna de las soluciones ofrecidas, se debe contar con la opinión de un experto en la temática (operador de red) o emplear una técnica de toma de decisiones. Usualmente este tipo de función se utiliza cuando se requiere mejorar los aspectos técnicos de la red, con el menor nivel de inversión posible. Es allí donde aparecen los objetivos en conflicto.

El modelo matemático del problema, además de contar con la función objetivo, debe considerar las restricciones que lo representan. Estas tienen que ser evaluadas para cada posible solución del problema. Dentro de las restricciones técnicas en los SD se encuentran balances de potencia activa y reactiva, límites de voltajes, límites de potencia inyectada por la GD y capacidad de transporte en las líneas [35]. Vale la pena destacar que si alguna solución no cumple todo el conjunto de restricciones, debe ser descartada o, en su defecto, habrá que plantear una penalización dentro de la función objetivo.

\subsection{Métodos de optimización implementados para ubicar y dimensionar GD}

Al momento de integrar GD en los sistemas de distribución, hay que tener claro el escenario donde se trabajará. Los escenarios de prueba para la integración de GD parten de las diferentes combinaciones posibles entre ubicación, tamaño, tecnología y número de unidades de GD, que puede ser simple (una unidad) o múltiple (instalación de dos o más unidades de GD) [11], [33]. Según el tipo de escenario, se requiere una técnica binaria, continua o una combinación de ambas, para dar solución al modelo matemático descrito en la sección 2.1. En la literatura existen diversos métodos y técnicas [32], [36], cuya clasificación y algunos estudios realizados se muestran a continuación.

\subsubsection{Métodos analíticos}

Utilizan un modelo matemático como la representación del sistema, evaluándolo con una solución numérica directa [37], la cual puede ser desarrollada por un software de optimización comercial. Ofrecen la ventaja de tener un tiempo computacional corto, son simples y precisos. Sin embargo, cuando el problema es complejo, su solución no es tan precisa. Diferentes autores han aplicado este método al problema de integración óptima de GD, como los que se presentan a continuación:

- Gözel y Hocaoglu [38] presentan una metodología para la ubicación y dimensionamiento $\mathrm{GD}$, a partir de un solo generador. Como función objetivo, fue 
seleccionada la minimización de las pérdidas totales de energía, y se evitó el uso de operaciones con la matriz de admitancias o la matriz jacobiana. El método fue probado en los sistemas IEEE de 12, 34 y 69 nodos, con una reducción máxima de las pérdidas para cada caso del $45,41 \%, 55,34 \%$ y $59,09 \%$, respectivamente. El método aplicado fue comparado con otras dos técnicas, y se concluyó que la respuesta obtenida por este era muy próxima a las otras.

- Gopiya et al. [39], en 2015, presentaron un enfoque analítico para la integración de GD, en busca de reducir las pérdidas de potencia activa y reactiva en la red. Su metodología inicia buscando el nivel de potencia activa y reactiva nodal que más beneficie a la red, seleccionando como nodos candidatos los que presentaron el mayor impacto positivo al sistema. Esta metodología se utiliza para ubicar uno o varios generadores y fue validada en los sitemas de 33 y 69 nodos, aplicando diferentes escenarios de prueba. Los mejores resultados presentaron una reducción del $69,55 \%$ y 89,89\%.

- Mahmoud et al. [35], en 2016, propusieron un método analítico para la ubicación óptima de múltiples unidades de GD y un flujo óptimo de potencias para su dimensionamiento; esto con el fin de reducir las pérdidas de energía. Se implementaron diferentes escenarios para la evaluación del impacto de GD. La técnica fue probada en los sistemas de 33 y 69 nodos, y se encontró como mejor solución entre los diferentes escenarios una reducción del $65,5 \%$ para el sistema de
33 nodos y del $71,56 \%$ para el sistema de 69 nodos.

Cabe resaltar que gran parte de los trabajos revisados para los métodos analíticos no examinan el impacto en los perfiles de tensión del sistema. Muchos autores no lo hacen debido a que las pérdidas de energía están estrechamente relacionadas con los perfiles de tensión nodal, de tal manera que si estas se reducen, los perfiles de tensión aumentarán. Se debe ser cuidadoso, pues en algunos casos los perfiles de tensión pueden superar los límites superiores establecidos por norma.

\subsubsection{Métodos numéricos}

También son llamados métodos basados en el cálculo, y se dividen en dos categorías: métodos directos e indirectos. Son restringidos a la hora de aplicarlos a problemas reales, debido a la alta complejidad que presentan; sin embargo, son eficientes en problemas sencillos. Para solucionar el problema de GD en el SD, los métodos más utilizados son [34]: programación lineal [40], programación no lineal [41], programación dinámica [42], programación entera [43] y programación estocástica [44].

Dentro de los diferentes trabajos existentes en la literatura se encuentra el realizado por Khoa et al. [45], que presenta un algoritmo basado en el método primario de doble punto interior para resolver problemas no lineales del flujo de potencia óptimo. El objetivo principal es optimizar la ubicación y el tamaño de la GD para resolver el problema de la reducción de las pérdidas de energía. Para validar su metodología implementan un 
sistema de 10 y 42 nodos. Este artículo presenta sus resultados al analizar las pérdidas de cada línea.

En el estudio realizado por Kaur et al. [46] inicialmente se preseleccionan los nodos candidatos a partir de un modelo de pérdida de sensibilidad combinado (Combined Loss Sensitivity [CLS]). Para la integración óptima (ubicación y dimensionamiento), se utilizan la técnica de Programación Secuencial Cuadrática (Sequential Quadratic Programming [SQP]) y el algoritmo de ramificación y acotación (Branch and bound [B\&B]). Se utiliza como objetivo la minimización de pérdidas del sistema, a partir de las cargas pico. La metodología propuesta fue probada en los sistemas de 33 y 69 nodos, y comparada con las técnicas de flujo de carga exhaustivo (Exhaustive Load Flow [ELF]), mejora analítica (Improved Analytical [IA]) y Particle Swarm Optimization (PSO). En el sistema de 33 nodos, se logra una reducción de pérdidas del $86,1 \%$, y se integran dos unidades de GD, con el valor de potencia inyectada mínima (2765 MVA). Para el sistema de 69 nodos, el método propuesto muestra un mejor rendimiento al momento de integrar múltiples unidades de GD (con inyección de potencia activa y reactiva), con una reducción de pérdidas del $98,1 \%$.

Keane et al. [47] desarrollan una metodología basada en programación lineal para determinar la ubicación y el dimensionamiento óptimos de GD con respecto a restricciones de tipo técnico. Se utiliza la interdependencia de los nodos con respecto al conjunto de restricciones. El objetivo es maximizar la generación entregada desde GD, teniendo en cuenta las siguien- tes restricciones: corriente nominal de las líneas, capacidad de transformadores, niveles máximos de corriente de cortocircuito en equipos, límites de tensión en los nodos, límites de energías renovables disponibles, entre otras. La metodología fue probada en una sección de la red de distribución de Irlanda, compuesta por alimentador (38/110 kV) y cinco nodos. Se evaluaron dos escenarios, el primero plantea la inyección desde el nodo más alejado del alimentador. Se parte de un valor inicial de capacidad de GD instalada. La máxima capacidad instalada de GD es de 11.20MW, ubicada en los nodos tres y seis. Para el segundo escenario, sin predeterminar una ubicación y tamaño iniciales, se tiene una generación total de GD de 22.74MW, distribuida en los cinco nodos del sistema. En esta metodología no se tiene en cuenta como función objetivo las pérdidas del sistema.

\subsubsection{Métodos heurísticos y metaheurísticos}

Hacen parte del conjunto de métodos no exactos, que a pesar de no poseer mecanismos, que garanticen la obtención de la solución óptima global permiten conseguir buenas soluciones para problemas de optimización de la vida real, con esfuerzos computacionales aceptables. El esfuerzo computacional involucra dos aspectos importantes: el tiempo de cálculo y la cantidad de memoria requerida en el proceso. Una heurística es un procedimiento simple basado en la experiencia, en el buen juicio o en la solución de modelos matemáticos reducidos, que permite encontrar soluciones de 
buena calidad para problemas complejos del tipo no polinomiales completos [48].

Los métodos heurísticos son lo más simples y de bajo nivel, y esto hace que puedan caer en óptimos locales, y alejarse así de las buenas soluciones. Por esta razón, en la actualidad son utilizados como punto de inicio de las metaheurísticas o para reducir el espacio de solución por medio del análisis de indicadores de sensibilidad, dado que permiten identificar y seleccionar el conjunto de atributos de mayor impacto para un problema y descartar los demás. Los indicadores de sensibilidad mas utilizados por las técnicas heurísticas para integración de GD son las sobrecargas en las líneas, los límites de tensión nodal, el índice de estabilidad de tensión, entre otros [12], [13], [49].

Las técnicas metaheurísticas guían y modifican las operaciones de heurísticas subordinadas para producir soluciones de alta calidad de manera eficiente, empleando estrategias de búsqueda exitosas y algoritmos bioinspirados. Algunas de las técnicas mono-objetivo más empleadas son: algoritmo genético [50], enjambre de partículas [51], recocido simulado [52], búsqueda tabú [53], algoritmos inmunes [54], colonia de hormigas [55], enjambre de abejas [56], entre otros. Dentro las técnicas multi-objetivo, las más conocidas y empleadas son: algoritmo de ordenamiento no dominado (NSGA, por sus siglas en inglés) [57] y el algoritmo caótico artificial colonia de abejas (CABC, de sus siglas en inglés) [58]. En la literatura se puede encontrar gran cantidad de trabajos que implementan este tipo de técnicas en la integración de GD, entre los que se encuentran los siguientes:
- De Sousa et al. [59] implementan un indicador de sensibilidad para la reducción de nodos candidatos a ubicación de GD, luego aplican un algoritmo genético (Genetic Algotihm [GA]) para la ubicación y dimensionamiento de GD, para lo cual tienen en cuenta múltiples generadores. La estrategia es evaluada en un sistema de prueba de 70 nodos y una red de 2678 nodos de una empresa de distribución en Brasil. Como función objetivo tienen en cuenta las pérdidas de energía, implementando una curva de demanda horaria. Los autores muestran la aplicación de la metodología al caso de 70 nodos, pero no indican la reducción del nivel de pérdidas de energía y la mejora en los perfiles de tensión. Para el caso del sistema real logran una reducción máxima de pérdidas del $47 \%$, y de esta manera realizan un amplio análisis sobre el impacto de la metodología en los perfiles de tensión del sistema.

- Kansal et al. [60] presentan una metodología que permite integrar tres tipos diferentes de GD en el SD, implementando como función objetivo las pérdidas de energía eléctrica. Emplean como técnica de solución el algoritmo PSO. Su trabajo es evaluado a través del uso de los sistemas de prueba de 33 y 69 nodos, con lo cual se logra una reducción máxima en las pérdidas de energía del $67,79 \%$ y del $89,69 \%$, respectivamente. Adicionalmente, analizan el impacto en los perfiles de tensión y comparan su técnica con otras como Colonia Artificial de Abejas (Artificial Bee Colony $[A B C])$ y $G A$, aplicadas en otras publica- 
ciones. Los niveles de potencia inyectados son superiores al del nodo slack en el caso base.

- En [55], los autores aplican la técnica de Optimización de Colonia de Hormigas (Ant Colony Optimization [ACO]) para la integración optima de GD, buscando la reducción en las pérdidas de energía. La metodología fue validada en los sistemas IEEE de 12 y 15 nodos, con pérdidas de energía del $84,66 \%$ y el $75,68 \%$, respectivamente. Aquí no se realiza un análisis en el impacto en los perfiles de tensión, y se implementa un único generador con una inyección de potencia activa cercana al valor de la inyectada por el nodo slack.

- Mohandas et al. [58] presentan una metodología para la integración de múltiples unidades de GD, utilizando el Indice de Rendimiento Multi-objetivo (Multi Objective Performance Index [MOPI]), para mejorar la estabilidad de la tensión del SD. El problema es resuelto implementando el algoritmo Caótico Artificial de Colonia de Abejas (Caotic Artificial Bee Colony [CABC]). La eficacia del algoritmo propuesto se valida mediante el ensayo de los sistemas de prueba de 38 y 69 nodos. En este trabajo, se considera únicamente inyección de potencia activa por parte de los GD, el modelo de carga de potencia constante, y se plantean escenarios con otros modelos de carga dependientes de la tensión, como el industrial, residencial y comercial. Con esto se presentan mejoras en los índices de tensión para los diferentes escenarios de prueba. Dentro de los casos analizados anteriormente, los autores no tienen en cuenta el costo de los GD.

\subsubsection{Métodos híbridos}

Los métodos híbridos permiten potenciar las técnicas metaheurísticas mediante la combinación de dos o más de estas, para la búsqueda de la solución de un problema. Estos métodos brindan una solución de buena calidad, cercana o igual a la óptima. Son muy implementados debido a las características del problema aquí tratado, dado que la ubicación presenta una característica binaria y el dimensionamiento una continua. En la mayoría de los documentos analizados en la sección anterior, se implementa una técnica metaheurística para la ubicación y un flujo óptimo para el dimensionamiento de los generadores. Esto hace que el dimensionamiento esté atado a la función objetivo del programa que aplica el flujo, que por lo general tiene en cuenta solo la reducción de pérdidas de energía, lo que puede limitar la búsqueda a óptimos locales. Dentro de los trabajos que se encuentran en la literatura que aplican métodos híbridos, se encuentra los siguientes:

- Grisales et al. en [15] implementan un GA para la ubicación y el algoritmo PSO para el dimensionamiento de tres tipos diferentes de tecnologías de GD. Presentan una función multi-objetivo ponderada, a partir de las pérdidas de energía, el error cuadrático de tensión y los costos de los generadores. Para validar la metodología se evaluaron los sistemas de 33 y 69 nodos, con una reducción de las pérdidas de energía del $56,77 \%$ y el 
$63,54 \%$, y del error cuadrado de tensión del $80,49 \%$ y $82,08 \%$. Se presenta un nivel de inversión para cada uno de los casos de USD\$1993667 y USD\$2386349, respectivamente.

- Kefayat et al. [56] presentan un híbrido entre los algoritmos $A C O$ y $A B C$, el primero encargado de la ubicación y el segundo del dimensionamiento de los GD, lo que permite instalar desde uno hasta siete generadores eólicos en el sistema. Se implementó una función multi-objetivo ponderada, que busca la reducción de potencia activa y reactiva, emisiones de $\mathrm{CO}_{2}$, costos de inversión y mejora del índice de estabilidad de tensión. Se tiene en cuenta la incertidumbre de la generación eólica y la carga. Para validar la metodología se implementaron los sistemas de 33 y 69 nodos, con diferentes valores en los pesos de ponderación de la función objetivo; con esto se obtuvieron diversos escenarios de prueba para cada caso. Como mejor solución se logró una reducción del $69,90 \%$ para las pérdidas de energía en el sistema de 33 nodos con una instalación de siete generadores y una de reducción del $71,33 \%$ para el sistema de 69 nodos instalando tres generadores. Dentro de las tablas mostradas por los autores se puede analizar el impacto en las otras funciones objetivo; en todos se obtuvo impactos positivos.

- Kansal et al. [61] presentan un enfoque híbrido para la ubicación de múltiples unidades de GD e implementan diferentes tipos de inyección de potencia al sistema. Para esto se utilizó la técnica de optimización PSO y un método analítico para el dimensionamiento de los generadores. Como función objetivo, presenta la reducción de pérdidas de energía en el SD. La técnica fue validada en los sistemas de 33 y 69 nodos, con lo que se logra una reducción máxima entre los diferentes escenarios planteados del $94,45 \%$ y $98,1 \%$, respectivamente; se presentan niveles de penetración de GD demasiados altos, por encima de la potencia generada por el nodo slack. Adicional a esto, los autores demuestran que se obtienen mejoras en los perfiles de tensión y factor de potencia del siste$\mathrm{ma}$, de forma indirecta.

Se puede concluir a partir del análisis realizado, para los métodos y técnicas de optimización planteados, que las funciones objetivo más implementadas en la literatura son la reducción de pérdidas de energía y la mejora de los perfiles de tensión. Por lo general, no se incluyen los costos de inversión y operación de los generadores, hecho que impide realizar un análisis de la recuperación de la inversión en un plazo determinado. Vale la pena destacar la dificultad para recuperar la inversión a corto plazo en un proyecto GD, solo teniendo en cuenta la reducción de pérdidas de energía. Se recomienda analizar los incentivos económicos para la integración de este tipo de tecnologías en el SD, dados al mejoramiento de las condiciones operativas y la disminución de $\mathrm{CO}_{2}$, ofrecidos por los entes reguladores de cada país.

La presentación de los tiempos de cómputo no se realiza en la mayoría de los documentos, porque cuando se realiza la planeación de un sistema de distribución, este se hace para un periodo largo (de cin- 
co a diez años). Esto hace que el tiempo de cómputo no sea una variable crítica para la mayoría de los autores, pues estos análisis no se realizan en tiempo real. En los casos que se indican, es utilizado para comparar la eficiencia entre diferentes técnicas de optimización. Cabe decir que se debe ser cuidadoso al momento de comparar, dado que entre una técnica y otra se encuentra una amplia diferencia entre su complejidad y espacio de solución analizado.

\section{INVESTIGACIONES FUTURAS}

Las tendencias en investigación para resolver el problema de ubicación y dimensionamiento óptimo de GD apuntan a las siguientes temáticas:

- Planificación coordinada: asociada a la reconfiguración de la red a través de la ubicación simultánea de GD, condensadores, dispositivos de protección y almacenadores de energía, considerando un determinado tiempo, en busca de mejorar las condiciones operativas del sistema [62]-[66].

- Introducción de incertidumbres al modelo matemático: parten del conocimiento de cómo se comporta el sistema e incluyen parámetros inciertos, como generación por fuentes renovables, variación de la carga y precios de la energía [67]. Con lo anterior se busca un modelo que represente de manera más realista el comportamiento estocástico del SD.

- Gestión activa de la red: referente a la operación activa del sistema de distribución, lo que implica la inversión en sis- temas de comunicación y control que permitan obtener información en tiempo real sobre la operación de la red y de las unidades de GD que se encuentran conectadas [68], [69].

- Métodos de optimización: los nuevos desarrollos apuntan a la creación de híbridos entre las diferentes técnicas aquí tratadas, de manera que se permitan resolver el problema de ubicación (binario) y dimensionamiento (continuo), con el mínimo esfuerzo computacional (memoria y tiempo) [70].

\section{CONCLUSIONES}

La integración de GD puede verse como una ayuda al sistema de distribución desde dos tópicos: permite suplir el aumento de la demanda, utilizando los activos existentes, y además puede impactar de forma positiva las condiciones operativas de la red, siempre y cuando se realice una adecuada ubicación y dimensionamiento de los generadores. Para su integración se debe tener en cuenta la necesidad técnica o económica por parte del OR o agente interesado, el modelo matemático del problema y la técnica de optimización por implementar. La interpretación de los resultados obtenidos depende del modelo de la carga utilizado, puesto que, según el modelo aplicado, se estará analizando una hora específica o un panorama del tiempo.

Al momento de integrar GD se deben considerar, además de los aspectos técnicos, los costos asociados. Dentro de los artículos aquí revisados se encontró que gran parte de los autores se enfoca solamente en los temas técnicos de la red, sin importar el 
costo que tiene asociado a la integración de GD (inversión y operación), ni los incentivos económicos brindados por los entes reguladores. Ambos contextos son importantes al momento de evaluar la tasa de inversión de retorno, que en muchos casos puede definir la viabilidad de un proyecto.

Muchos autores proponen inyectar altos niveles de potencias a través de GD, a veces por encima de la potencia entregada por el generador principal en el caso base ( $\sin \mathrm{GD}$ ). Esto hace que las soluciones entregadas por sus metodologías no puedan ser llevadas a una aplicación real. Por tanto, se debe restringir la capacidad máxima de GD instalada en el SD, a la potencia demanda más las pérdidas del sistema. Adicional a esto, es importante realizar un estudio de la capacidad de generación de energía por cada tipo de tecnología de GD ubicada en el sistema.

Los sistemas de prueba más utilizados fueron el de 33 y 69 nodos, puesto que presentan altos niveles de pérdidas de energía y violaciones tensiones nodales; además, permiten apreciar con mayor claridad el impacto de las metodologías empleadas.

Al realizar una revisión del estado del arte sobre la integración de GD, se encontró que los escenarios de prueba nacen de la combinación de su ubicación y dimensionamiento, utilizando como la mejor alternativa el análisis de múltiples unidades de GD. Por lo general, el tipo de carga modelado es mono-nivel y las funciones objetivo más utilizadas son la disminución de pérdidas de energía y las mejoras en los perfiles de tensión, siendo las técnicas de optimización metaheurísticas las más implementadas en los últimos años.

\section{REFERENCIAS}

[1] P.P. Barkery R. W. de Mello, “Determining the impact of distributed generation on power systems. I. Radial distribution systems," en Power Engineering Society Summer Meeting (Cat. No.00CH37134), vol. 3, pp. 1645-1656, 2000. https://doi. org/10.1109/PESS.2000.868775

[2] E. K. Bawan, "Distributed Generation Impact on Power System Case study: Losses and Voltage Profile," en 2012 22nd Australasian Universities Power Engineering Conference (AUPEC), pp. 1-12, 2012.

[3] P. Prakash y D. K. Khatod, "Optimal sizing and siting techniques for distributed generation in distribution systems: A review," Renewable and Sustainable Energy Reviews, vol. 57, pp. 111-130, 2016. https://doi.org/10.1016/j.rser.2015.12.099

[4] A. J. Bazurto Cubillos et al., "Perspectiva del transformador de distribución en redes eléctricas con alta penetración de generación distribuida y vehículos eléctricos," Ciencia e Ingeniería Neogranadina, vol. 26, no. 2, pp. 35-48, 2016. https://doi.org/10.18359/rcin.1710

[5] M. Barnes et al., "Real-World MicroGrids-An Overview," en IEEE International Conference on System of Systems Engineering, pp. 97-105, San Antonio, TX, Estados Unidos, 16-18 Abril 2007. https://doi.org/10.1109/sysose.2007.4304255

[6] N. Hatziargyriou et al., "Microgrids," IEEE Power and Energy Magazine, 
vol. 5, no. 4, pp. 78-94, 2007. https:// doi.org/10.1109/MPAE.2007.376583

[7] S. Morozumi, "Micro-grid Demonstration Projects in Japan," en 2007 Power Conversion Conference, pp. 635-642, Nagoya, Japan, 2-5 Abril, 2007. https:// doi.org/10.1109/PCCON.2007.373032

[8] Congreso de Colombia, "Ley N ${ }^{\circ} 1715$ del 13 de mayo de 2014," UPME. [En Linea]. Disponible en: http://wsp.presidencia.gov.co/Normativa/Leyes/ Documents/LEY\%201715\%20DEL\%20 13\%20DE\%20MAYO\%20DE\%202014. pdf

[9] Unidad de Planeación Minero Energética [UPME]. Integración de las energías renovables no convencionales en Colombia. Bogotá: La Imprenta Editores S.A, 2015.

[10] N. Jenkins, J.B. Ekanayake and G. Strbac. Distributed Generation. London, United Kingdom: The Institution of Engineering and Technology, 2010. https://doi.org/10.1049/pbrn001e

[11] M. Kumawat et al., "Optimal distributed generation placement in power distributed networks: A review," en 2015 International Conference on Electrical, Electronics, Signals, Communication and Optimization (EESCO), Visakhapatnam, India, 24-25 Ene. 2015. https://doi.org/10.1109/EESC0.2015.7254023

[12] I. F. Prado y L. P. Garces, "Chu-Beasley genetic algorithm applied to the allocation of distributed generation," en 2013 IEEE PES Conference on Innovative Smart Grid Technologies (ISGT Latin
America), pp. 1-7, Sao Paulo, Brazil, 1517 Abr. 2013. https://doi.org/10.1109/ ISGT-LA.2013.6554425

[13] M. Sedighi et al., "Sitting and sizing of distributed generation in distribution network to improve of several parameters by PSO algorithm," en 2010 9th International Power and Energy Conference, IPEC 2010, pp. 10831087, Singapore, Singapore, 27-29 Oct. 2010. https://doi.org/10.1109/ IPECON.2010.5696977

[14] B. Venkatesh et al., "Optimal reconfiguration of radial distribuion system using artificial intelligence methods," en 2009 IEEE Toronto International Conference Science and Technology for Humanity (TIC-STH), pp. 660-665, Toronto, ON, Canada, 26-27 Sept. 2009. https://doi. org/10.1109/TIC-STH.2009.5444417

[15] L. F. Grisales et al., "Optimal location and sizing of Distributed Generators using a hybrid methodology and considering different technologies," 2015 IEEE 6th Latin American Symposium on Circuits \& Systems (LASCAS), Montevideo, Uruguay, 24-27 Feb. 2015. https://doi. org/10.1109/LASCAS.2015.7250486

[16] M. B. Jannat y A. S. Savić, "Optimal capacitor placement in distribution networks regarding uncertainty in active power load and distributed generation units production," IET Generation, Transmission \& Distribution, vol. 10, pp. 3060-3067, 2016. https://doi. org/10.1049/iet-gtd.2016.0192

[17] J. Posso et al., "Planeamiento de redes de baja tensión, utilizando un modelo 
trifásico," Ciencia e Ingeniería Neogranadina, vol. 21, no. 2, pp. 41-56, 2011. https://doi.org/10.18359/rcin.259

[18] D. D. Lucache et al., "Economic apraisal of optimal location and sizing of capacitors in radial distribution systems using PSO," en 2014 International Conference on Applied and Theoretical Electricity (ICATE), Craiova, Romania, 23-25 Oct. 2014. https://doi.org/10.1109/ ICATE.2014.6972626

[19] S. Yoshizawa et al., "Novel voltage control of multiple step voltage regulators in a distribution system," en 2014 IEEE PES on Innovative Smart Grid Technologies Conf. (ISGT), Washington, D.C., Estados Unidos, 19-22 Feb. 2014. https:// doi.org/10.1109/ISGT.2014.6816463

[20] T. Ackermann et al., "Distributed generation: a definition," Electric Power Systems Research, vol. 57, pp. 195-204, 2001.

[21] T. Ackermann, "What Matters for Successful Integration of Distributed Generation Energynautics Areas of Expertise," en Future Challenges for Electricity Security - IEA Workshop, Paris, France, 1 Oct. 2013. [En línea]. Disponible en: https://www.iea.org/workshops/futurechallenges-for-electricity-security.html

[22] International Conference on Large Electric Systems [CIGRE], "SC C6 Distribution Systems and Dispersed Generation," s. f. [En línea]. Disponible en: http://www.cigre.org/Technical-activities/Study-Committees-Working-Groups/SC-C6-Distribution-Systems-and-Dispersed-Generation
[23] A. Rezaee Jordehi, "Allocation of distributed generation units in electric power systems: A review," Renewable and Sustainable Energy Reviews, vol. 56, pp. 893-905, 2016. https://doi. org/10.1016/j.rser.2015.11.086

[24] K. Balamurugan et al., "Impact of Distributed Generation on Power Distribution Systems," Energy Procedia, vol. 25, pp. 93-100, 2012. https://doi. org/10.1016/j.egypro.2012.07.013

[25] V. V. S. N. Murty y A. Kumar, "Optimal placement of $D G$ in radial distribution systems based on new voltage stability index under load growth," International Journal of Electrical Power \& Energy Systems, vol. 69, pp. 246256, 2015. https://doi.org/10.1016/j. ijepes.2014.12.080

[26] Z. Sun y X. Zhang, "Advances on Distributed Generation Technology," Energy Procedia, vol. 17, pp. 32-38, 2012. https://doi.org/10.1016/j.egypro.2012.02.058

[27] A. Yadav y L. Srivastava, "Optimal placement of distributed generation: An overview and key issues," en 2014 International Conference on Power Signals Control and Computations (EPSCICON), Thrissur, India, 6-11 Ene. 2014. https://doi. org/10.1109/EPSCICON.2014.6887517

[28] J. A. Hernandez et al., "Analysis of the effect of the implementation of photovoltaic systems like option of distributed generation in Colombia," Renewable and Sustainable Energy Reviews, vol. 15, pp. 2290-2298. https://doi. org/10.1016/j.rser.2011.02.003 
[29] J. Grainger y W. Stevenson. Power System Analysis. Nueva York, Estados Unidos: McGraw-Hill, 1994.

[30] S. Ghosh et al., "Optimal sizing and placement of distributed generation in a network system," International Journal of Electrical Power \& Energy Systems, vol. 32, pp. 849-856. https://doi. org/10.1016/j.ijepes.2010.01.029

[31] S. Kansal et al., "Optimal placement of different type of DG sources in distribution networks," International Journal of Electrical Power and Energy Systems, vol. 53, pp. 752-760. https://doi. org/10.1016/j.ijepes.2013.05.040

[32] R. A. Gallego et al. Técnicas metaheurísticas de optimización (2da. Ed.). Pereira, Colombia: Universidad Tecnológica de Pereira, 2008.

[33] P. S. Georgilakis y N. D. Hatziargyriou, "Optimal Distributed Generation Placement in Power Distribution Networks: Models, Methods, and Future Research," IEEE Transactions on Power Systems, vol. 28, pp. 3420-3428. https://doi.org/10.1109/ TPWRS.2012.2237043

[34] S. S. Rao. Engineering Optimization: Theory and Practice (4ta. Ed.). Hoboken, NJ. Estados Unidos: John Wiley \& Sons, Inc., 2009.

[35] K. Mahmoud et al., "Optimal Distributed Generation Allocation in Distribution Systems for Loss Minimization," IEEE Transactions on Power Systems, vol. 31, pp. 960-969, 2016. https://doi. org/10.1109/TPWRS.2015.2418333
[36] S. P. Karthikeyan et al., "A review on soft computing techniques for location and sizing of distributed generation systems," en 2012 International Conference on Computing, Electronics and Electrical Technologies (ICCEET), pp. 163-167, Kumaracoil, India, 21-22 Mar. 2012. https://doi.org/10.1109/ICCEET.2012.6203882

[37] C. Wang y M. H. Nehrir, "Analytical Approaches for Optimal Placement of Distributed Generation Sources in Power Systems," IEEE Transactions on Power Systems, vol. 19, pp. 20682076, 2004. https://doi.org/10.1109/ tpwrs.2004.836189

[38] T. Gözely M. H. Hocaoglu, "An analytical method for the sizing and siting of distributed generators in radial systems," Electric Power Systems Research, vol. 79, pp. 912-918, 2009. https://doi.org/10.1016/j.epsr.2008.12.007

[39] S. N. Gopiya Naik et al., "Analytical approach for optimal siting and sizing of distributed generation in radial distribution networks," IET Generation, Transmission \& Distribution, vol. 9, pp. 209-220, 2015. https://doi. org/10.1049/iet-gtd.2014.0603

[40] B. Singh et al., "A survey on impact assessment of DG and FACTS controllers in power systems," Renewable and Sustainable Energy Reviews, vol. 42, pp. 846-882, 2015. https://doi.org/10.1016/j.rser.2014.10.057

[41] S. Porkar et al., "Optimal allocation of distributed generation using a two-stage multi-objective mixed-integer-nonlinear 
programming," European Transactions on Electrical Power, vol. 21, pp. 10721087, 2011. https://doi.org/10.1002/ etep.497

[42] N. Khalesi et al., "DG allocation with application of dynamic programming for loss reduction and reliability improvement," International Journal of Electrical Power \& Energy Systems, vol. 33, pp. 288-295, 2011. https://doi.org/10.1016/j.ijepes.2010.08.024

[43] J. D. Foster et al., "Comparison of Mixed-Integer Programming and Genetic Algorithm Methods for Distributed Generation Planning," IEEE Transactions on Power Systems, vol. 29, pp. 833-843, 2014. https://doi. org/10.1109/TPWRS.2013.2287880

[44] Z. Zhou et al., "A two-stage stochastic programming model for the optimal design of distributed energy systems," Applied Energy, vol. 103, pp. 135-144. https://doi.org/10.1016/j.apenergy.2012.09.019

[45] T. Q. D. Khoa et al., "Optimizing Location and Sizing of Distributed Generation in Distribution Systems," en 2006 IEEE PES Power Systems Conference and Exposition, pp. 725-732, Atlanta, GA, Estados Unidos, 29 Oct.-1 Nov. 2006. https://doi.org/10.1109/ PSCE.2006.296407

[46] S. Kaur et al., "A MINLP technique for optimal placement of multiple DG units in distribution systems," International Journal of Electrical Power and Energy Systems, vol. 63, pp. 609-617, 2014. https:// doi.org/10.1016/j.ijepes.2014.06.023
[47] A. Keane y M. O'Malley, "Optimal Allocation of Embedded Generation on Distribution Networks," IEEE Transactions on Power Systems, vol. 20, pp. 16401646, 2015. https://doi.org/10.1109/ TPWRS.2005.852115

[48] R. Gallego et al. Técnicas metaheurísticas de optimización. Pereira, Colombia: Universidad Tecnológica de Pereira, 2008.

[49] D. N. Hussein et al., "Optimal Sizing and Siting of Distributed Generation," en International Middle East Power System Conference, 2006.

[50] P. C. Chu y J. E. Beasley, "A genetic algorithm for the generalised assignment problem," Computers \& Operations Research, vol. 24, no. 1 pp. 17-23, 1997. https://doi.org/10.1016/S03050548(96)00032-9

[51] M. T. A. Y. Mohammadi y M. Faramarzi, "PSO algorithm for Sitting and Sizing of Distributed Generation to Improve Voltage Profile and Decreasing power losses," en Procedings of 17th Conference on Electrical Power Distribution Networks (EPDC), Tehran, Iran, 2-3 May, 2012.

[52] A. K. Barnes et al., "Placement of energy storage coordinated with smart PV inverters," en 2012 IEEE PES Innovative Smart Grid Technologies (ISGT), Washington, D.C., Estados Unidos, 16-20 Ene. 2012. https://doi.org/10.1109/ ISGT.2012.6175665

[53] D. Zhang et al., "An improved TS algorithm for loss-minimum reconfiguration in large-scale distribution systems," 
Electric Power Systems Research, vol. 77, pp. 685-694, 2007 https://doi. org/10.1016/j.epsr.2006.06.005

[54] M. Junjie et al., "Size and Location of Distributed Generation in Distribution System Based on Immune Algorithm," Systems Engineering Procedia, vol. 4, pp. 124-132, 2012. https://doi. org/10.1016/j.sepro.2011.11.057

[55] B. Sookananta et al., "Determination of the optimal location and sizing of Distributed Generation using Ant Colony Search," en 2010 International Conference on Electrical Engineering/Electronics Computer Telecommunications and Information Technology (ECTI-CON), Chiang Mai, Thailand, 19-21 May. 2010.

[56] M. Kefayat et al., "A hybrid of ant colony optimization and artificial bee colony algorithm for probabilistic optimal placement and sizing of distributed energy resources," Energy Conversion and Management, vol. 92, pp. 149-161, 2015. https://doi.org/10.1016/j.enconman.2014.12.037

[57] M. Moeini-Aghtaie et al., "Optimal Distributed Generation placement in a restructured environment via a multi-objective optimization approach," en 16th Electrical Power Distribution Conference, Bandar Abbas, Iran, 19-20 Abril 2011.

[58] N. Mohandas et al., "Optimal location and sizing of real power DG units to improve the voltage stability in the distribution system using $A B C$ algorithm united with chaos," International Journal of Electrical Power Energy Systems, vol. 66, pp. 41-52, 2015.
[59] A. R. R. de Sousa et al., "Sensitivity analysis to connect distributed generation," International Journal of Electrical Power Energy Systems, vol. 46, pp. 145152, 2013. http://dx.doi.org/10.1016/j. ijepes.2012.10.004

[60] S. Kansal et al., "Optimal placement of different type of DG sources in distribution networks," International Journal of Electrical Power and Energy Systems, vol. 53, pp. 752-760, 2013. https://doi. org/10.1016/j.ijepes.2013.05.040

[61] S. Kansal et al., "Hybrid approach for optimal placement of multiple DGs of multiple types in distribution networks," International Journal of Electrical Power Energy Systems, vol. 75, pp. 226235, 2016. https://doi.org/10.1016/j. ijepes.2015.09.002

[62] A. Bayat et al., "Optimal siting and sizing of distributed generation accompanied by reconfiguration of distribution networks for maximum loss reduction by using a new UVDA-based heuristic method," International Journal of Electrical Power \& Energy Systems, vol. 77, pp. 360-371, 2016. https://doi. org/10.1016/j.ijepes.2015.11.039

[63] A. M. Tahboub et al., "Distribution System Reconfiguration for Annual Energy Loss Reduction Considering Variable Distributed Generation Profiles," IEEE Transactions on Power Delivery, vol. 30, pp. 1677-1685, 2015. https://doi. org/10.1109/TPWRD.2015.2424916

[64] F. Ding y K. A. Loparo, "Feeder Reconfiguration for Unbalanced Distribution Systems With Distributed Generation: A 
Hierarchical Decentralized Approach," IEEE Transactions on Power Systems, vol. 31, pp. 1633-1642, 2016. https://doi. org/10.1109/TPWRS.2015.2430275

[65] S. W. Alnaser y L. F. Ochoa, "Optimal Sizing and Control of Energy Storage in Wind Power-Rich Distribution Networks," IEEE Transactions on Power Systems, vol. 31, pp. 2004-2013, 2016. https://doi. org/10.1109/TPWRS.2015.2465181

[66] A. Saif et al., "Optimal allocation of distributed energy resources through simulation-based optimization," Electric Power Systems Research, vol. 104, pp. 1-8, 2013. https://doi.org/10.1016/j. epsr.2013.05.019

[67] S. Ganguly y D. Samajpati, "Distributed Generation Allocation on Radial Distribution Networks Under Uncertainties of Load and Generation Using Genetic Algorithm," IEEE Transactions on Sustainable Energy, vol. 6, pp. 688-
697, 2015. https://doi.org/10.1109/ TSTE.2015.2406915

[68] V. F. Martins C. L. T. Borges, "Active distribution network integrated planning incorporating distributed generation and load response uncertainties," IEEE Transactions on Power Systems, vol. 26, pp. 2164-2172, 2011. https://doi. org/10.1109/TPWRS.2011.2122347

[69] K. Zare et al., "Dynamic planning of distributed generation units in active distribution network," IET Generation, Transmission \& Distribution, vol. 9, pp. 1455-1463, 2015. https://doi. org/10.1049/iet-gtd.2014.1143

[70] M. Vatani et al., "Multiple distributed generation units allocation in distribution network for loss reduction based on a combination of analytical and genetic algorithm methods," IET Generation, Transmission \& Distribution, vol. 10, pp. 66-72, 2016. https://doi. org/10.1049/iet-gtd.2015.0041 